\title{
Italique
}

Poésie italienne de la Renaissance

XVIII | 2015

Varia

\section{Il dittico tragico di compassione e orrore nella Adriana e nella Dalida di Luigi Groto}

\section{Bernhard Huss}

Traduttore: Maraike Di Domenica

\section{OpenEdition}

\section{Journals}

Edizione digitale

URL: http://journals.openedition.org/italique/403

DOI: 10.4000/italique.403

ISSN: $1663-4438$

\section{Editore}

Librairie Droz

\section{Edizione cartacea}

Data di pubblicazione: 1 ottobre 2015

Paginazione: 35-61

ISBN: 978-2-600-01900-2

ISSN: 1423-3983

\section{Notizia bibliografica digitale}

Bernhard Huss, "Il dittico tragico di compassione e orrore nella Adriana e nella Dalida di Luigi Groto », Italique [Online], XVIII | 2015, online dal 01 octobre 2017, consultato il 03 mai 2019. URL : http:// journals.openedition.org/italique/403 ; DOI : 10.4000/italique.403 
B ER NHAR D H US

\section{L D I T T I C O TR A G I C O \\ D I C OMPASSIONE E ORRORE}

NELLA $A D R I A N A$ E NELLA $D A L I D A$

D I L U I I GROTO

trar dal petto vostro e da le ciglia un'Etna di sospiri, un mar di pianto

Groto, Adriana, Prologo, vv. 5-6 vi farò di horrore gelar le vene, \& arricciar le chiome

Groto, La Dalida, 4.I 



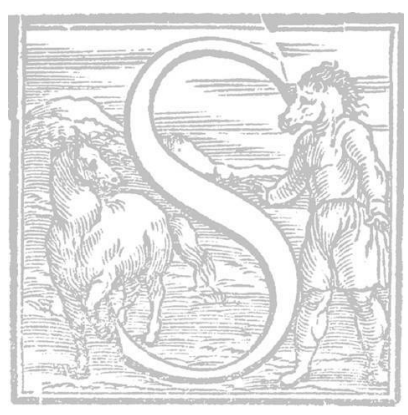

olo in pochi, senz'altro esperti di letteratura del Rinascimento italiano e di manierismo letterario, ${ }^{\mathrm{I}}$ ricordano il nome di Luigi Groto (I54I-I585). Eppure, in vita, questo poeta è stato una delle figure di spicco dell'ambiente letterario del tardo Rinascimento; in un Veneto culturalmente molto attivo era conosciuto da tutti, e dopo la sua morte diventò addirittura «weit ins 17. Jahrbundert binein ein in ganz. Europa berühmter Mann». ${ }^{3}$ Redasse su commissione pubblica una serie di discorsi che furono declamati in occasioni politiche, scrisse inoltre numerose lettere, edite all'inizio del XVII secolo in tre raccolte, compose dialoghi e commenti (andati perduti) a testi scientifici (di astronomia, agricoltura e geologia), rielaborò e commentò $i$ Cinque canti dell'Ariosto e il Decamerone di Boccaccio. Oggi, per noi, la sua importanza storico-letteraria risiede soprattutto nelle vaste composizioni delle $\mathrm{Rime}^{4}$ e in una serie di testi teatrali, molto apprezzati all'epoca dell'autore (Dalida I572, Il pentimento amoroso 1576 , Adriana 1578 , Emilia 1579 , Il tesoro I580, Calisto I582). 'L'opera drammatica di Groto è stata poco studiata; l'unica eccezione è rappresentata dalla Adriana, pubblicata e interpretata di recente da Marco Ariani, ${ }^{6}$ che però è stata poi presa in considerazione quasi unicamente per capire se Shakespeare, durante la stesura di Romeo and Juliet, avesse presente o meno come modello il testo teatrale grotiano. ${ }^{7}$ Questa reticenza della ricerca non è assolutamente in sintonia con la ricezione coeva delle sue opere, piuttosto vivace, almeno considerando le numerose ristampe dei drammi. ${ }^{8}$ L'Adriana fu ristampata almeno sette volte, tra il I586 e il I619,9 la Dalida ha visto almeno sei ristampe tra il is86 e il I646. L'opera di Groto si distingue per l'interesse, classificabile come manierista, a sottoporre le capacità dei generi letterari da lui usati a una specie di "test di resistenza". Ciò è evidente, ad esempio, nelle Rime, nelle quali l'autore porta alla soglia di rottura le norme prescrittive del bembismo ortodosso. La dizione petrarchista e la topica petrarchesca vengono accentuate in maniera antitetica e ossimorica fino all'estremo. La divaricazione e la scomposizione manierista dei moduli lirici ciconducono ad un inesorabile sperimentalismo le possibilità e $i$ limiti dei sistemi letterari codificati. ${ }^{10}$ Il potenziale degli schemi dei generi letterari rispetto alle loro funzionalità 
viene esaminato da Groto in actu, anche per quanto riguarda la potenzialità del singolo genere, in caso di immissione di elementi "estranei" (come ad esempio l'immissione della tradizione epigrammatica nel petrarchismo lirico in lingua volgare). Groto scandaglia sistematicamente il campo dei generi esistenti nel Secondo Cinquecento. Per quanto concerne il dramma egli sottopone $i$ tre grandi registri disponibili - la tragedia, la commedia e il dramma pastorale - ai suoi "test"; con l'opera giovanile Isac (rappresentato nel I558 e nel I58 e stampato nel I5 86 e nel I605) si aggiunge anche un "dramma sacro". "I

La prova di resistenza letterario-pratica va sempre di pari passo alla abile e forzosa messa in scena della figura auctoris di Groto. ${ }^{{ }^{2}}$ Nelle Rime, per esempio, si può osservare come l'uso radicale delle pratiche poetiche petrarchiste metta in evidenza soprattutto la "maniera" dell'autore. La tendenza del testo lirico all'autoriflessione corrisponde qui alla messa in scena e al rispecchiamento dell'autore empirico nella sua figura testuale. A questo riguardo, sia nelle Rime che in altri testi, ritorna di frequente il tema della cecità come sofferenza personale e invalidante dell'autore, ${ }^{\mathrm{I}}$ in stretto legame con la tematica testuale della sofferenza e con il loro stile "tormentato", teso e aspro.

Entrambi gli aspetti - da un lato il vigoroso profilarsi dell'autore, dall'altro l'esperimento manierista attuato tramite $i$ procedimenti di composizione dei singoli generi - sono evidenti anche nelle tragedie di Groto, l'Adriana ${ }^{\mathrm{I}}$ e la Dalida. ${ }^{\mathrm{I}}$ Nel prologo dell'Adriana si asserisce che «l'autore» avrebbe trovato la «istoria» dei due protagonisti "scritta in duri marmi», e che avrebbe scoperto in un'annotazione l'incarico alla composizione di questa storia. Questo incarico gli avrebbe conferito il diritto alla dispositio tragica degli avvenimenti e anche alla necessaria estensione dell'unità temporale; inoltre avrebbe anticipato una giustificazione del suo prologo tragico, che non ha radici antiche, ma che era stato precedentemente impiegato in maniera eminente da Giraldi nell'Orbecche. ${ }^{16}$ Il risultato di questo incarico sarebbe il dramma stesso, che verrà presentato ai lettori come testo o agli spettatori sulla scena. Mediante la tecnica della metalessi, alla fine dell" "azione" del dramma, Adriana stessa esprimerà l'incarico rivolto ad un "autore" in tempi lontani: sarebbe stata proprio lei a esternare il desiderio di far incidere «in duri marmi» la sua infelice storia d'amore per indurre «qualche autor, mosso a pietà, negli anni / avvenim» a una riattualizzazione drammatico-teatrale ("la riduca in forma, ch'ella / possa 
rappresentarsi a' fidi amanti»). ${ }^{17}$ Luigi Groto si fa incaricare dai personaggi da lui stesso inventati della stesura della loro azione scenica. Questo "strange loop", che comporta una grave infrazione del concetto aristotelico di verosimiglianza in vigore all'epoca, non soltanto concede, all'interno del testo stesso, uno spazio tangibile ed empirico all'autore, ma anche un'ampia expertise, che deve essere letta alla luce del dibattito rinascimentale sulla (i) liceità di soggetti del tutto inventati (vs. soggetti "storicamente" accertati). Il profilo dell'autore viene in tal modo accentuato, e questo fa chiaramente risaltare il carattere letterario del testo dell'Adriana. Il tempo dell'azione viene collegato con il tempo della sua scrittura o della sua rappresentazione. Il tutto viene sorretto dall'identità dei luoghi dove si trovano attori e spettatori: il Prologo comunica al pubblico l'identità tra il luogo dell'azione drammatica e il suo ambiente empirico, con la differenza che, rispetto al tempo dell'azione, oggi la città di Adria sarebbe caratterizzata da una decadenza generale che avrebbe avuto inizio proprio con gli allagamenti (qui spiegati come sabotaggio bellico) verificatisi alla fine dell'azione drammatica. $^{\text {I }}$

Anche in Dalida la figura dell'autore viene introdotta in posizione centrale nel Prologo tragico, ${ }^{\text {I9 }}$ in ampia corrispondenza con la descrizione dolorosa della figura del Cieco d'Adria nelle Rime di Groto. Nello stesso momento in cui il Prologo descrive come tematica della tragedia terribili $\pi \dot{\alpha} \theta \eta$ ("lamenti [...], lacrime, e morti») e annuncia uno stile tipicamente "grotiano" per la composizione ("In meste strida, in tristo, \& aspro stile»), l'orrore delle sofferenze presentate nell'azione viene collegato alle terribili sofferenze della vita dell'autore: ${ }^{20}$ questi vive in una sfera del terrore, reagisce emotivamente con eccessi di sofferenza e rende, insieme all'azione della Dalida, la propria disgrazia oggetto delle sue esternazioni: "[...] l'Auttor nostro in tenebroso borrore / Con Heraclito ogn'bor vivrà piangendo / In meste strida, in tristo, \& aspro stile / Con le miserie altrui le proprie pene». Le «proprie pene» sono il risultato di un'esistenza scossa da colpi clamorosi, e da un accumulo di disgrazie ( $E E$ certo, ch'altro attender si potea / Da si misero Auttor? Deb Dio, che mentre / Ei sta piangendo una miseria sua, / Vn'altra sopr'arriva, e un'altra, e un'altra, / Si ch'ei s'arresta attonito, \& incerto / Qual prima debba piangere, e qual poi»). ${ }^{2 \mathrm{I}}$ L'autore, la cui storia viene raccontata dal Prologo come «historia calamitatum», ${ }^{22}$ è straziato da una terribile povertà; ${ }^{23}$ inoltre il des- 
tino lo ha duramente colpito fin da bambino con la sofferenza della cecità (il fatto che non si meritasse questo colpo fornisce al Prologo lo spunto per riflettere sulla fondamentale ingiustizia del mondo) ${ }^{24}$ da neonato ha perso il padre; in età scolastica ha perso il suo venerato maestro; ${ }^{25}$ in seguito ha perso «La carissima Madre», sicché «nulla di ben gli avanzi in terra»; ${ }^{26} e$ in ultimo ha perduto il suo cuore in un amore dai connotati petrarchisti per una dama presente nel pubblico, il cui nome però non può essere enunciato da chi recita il prologo. ${ }^{27}$ Tramite questo concettismo non solo l'azione drammatica carica di dolore viene avvicinata alle sofferenze del suo autore, ${ }^{28}$ ma anche, al contempo, alla situazione contingente della rappresentazione. Del resto anche nella Dalida (anche se in maniera meno ostentata dell'Adriana) si parte dall'azione per tematizzare con una metalessi il lavoro di scrittura dell'autore empirico Groto; è Candaule, inebriato dalla sete di vendetta contro Berenice, a incaricare uno scrittore tragico futuro della rappresentazione della potenza della sua vendetta: «Ma ti prometto ben, ma ben ti giuro, / Ch'io vò, che qualche tragico scrittore / Ne i secoli avvenir ponga in iscena / Vna nova Tragedia in sù l'essempio, / Che al mondo io lascierò de la vendetta». ${ }^{29}$ I diversi piani della comunicazione drammatica vengono sourapposti in maniera cosi evidente che l'effetto sembra di nuovo una letterarietà forzata dell'intero costrutto testuale della tragedia. Questo effetto viene ulteriormente incrementato attraverso un'altra metalessi che va a discapito di qualsiasi "verosimiglianza": parlando con Candaule, il suo Consigliere indica con gesto epidittico $e$ in lettere maiuscole le persone dell" "Illustrissima ALES$S A N D R A[\ldots]$ VOLTA», suo marito e i loro figli «ANTONIO» $e$ «ORSINA». ${ }^{3 \circ}$ Alessandra Volta, una protettrice di Groto realmente esistita, è anche la destinataria della dedica nella prefazione alla Dalida, e cosi il Consigliere riassume e raccoglie praticamente tutti $i$ piani testuali dell'edizione stampata dell'opera. L'autore, la sua opera, l'azione e la rappresentazione vengono portati alla coscienza in quanto conglomerato comunicativo, e cosi appare fin dall'inizio preclusa l'immedesimazione illusionistica nell" "azione" in quanto tale, che dal punto di vista aristotelico è garante delle passioni operative nel genere tragico.

La disgregazione delle due passioni di é̀ $\lambda \mathrm{s}_{\mathrm{s}}$ e póbos è dovuta, nel progetto della tragedia di Groto, alla sperimentazione con $i$ dispositivi che secondo la coscienza teorica dell'epoca erano a disposizione del 
genere tragico. Cosi come nella pastorale ha realizzato un dittico sperimentale (Calisto, un pezzo scabroso-satirico, Il pentimento amoroso, un pezzo di tipo comicizzante), ${ }^{3 \mathrm{I}}$ cosi rende evidente anche in ambito tragico un'antitesi e, attraverso un movimento che acuisce ancor di più la distanza tra le tragedie cinquecentesche più conosciute (come l'Orbecche di Giraldi e la Canace di Speroni) e $i$ testi delle tragedie dell'antichità, ${ }^{32}$ arriva a "due spericolate avventure formali significativamente lanciate in opposte direzioni». ${ }^{33}$ Diversamente dalla teoria del tempo, che considerava gli effetti emotivi di «compassione» e «spavento» come affini, anche se, seguendo il modello della Poetica di Aristotele (cap. I8), continuava a differenziare la forma della tragedia piu emotivamente accentuata, la "tragedia affettuosa» ( $\left.\pi \alpha \theta \eta \tau \iota x \eta^{\prime}\right)$ dalla «trage-

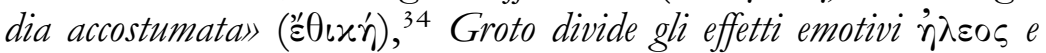
póbos e li distribuisce nel dittico tragico di Adriana come tragedia della "compassione" e Dalida come tragedia dell'orrore. Ciò non comporta alcun accostamento alla dottrina aristotelica, ${ }^{35}$ ma è all'insegna di un atteggiamento fondamentalmente manierista, come abbiamo potuto mostrare per le Rime: Groto si appropria di ogni possibilità rappresentativa, dei mezzi di effetto di un determinato registro tipico per un genere, e li declina fino alle estreme conseguenze. Secondo lo svolgimento dell'Orbecche, un'opera che aveva inaugurato il modello della tragedia dell'orrore ${ }^{36}$ e si era distinta in maniera radicale dalla tradizione delle tragedie razionalmente moderate avviata da Trissino con la Sofonisba, dove ci si voleva servire di una forma "aristotelica" per addomesticare le passioni, ${ }^{37}$ la tragedia dell'orrore e la tragedia della compassione erano distinguibili come sottogeneri di cui Groto metteva alla prova il rispettivo potenziale. ${ }^{38}$

Già nel Prologo l'Adriana si configura come tragedia della compassione; la poetica dell'opera sembra completamente fondata sull'’' $\lambda \varepsilon \circ \varsigma$ (pieta). Sin dal secondo verso del Prologo si vuole carpire negli occhi del pubblico un «pietoso umor», quel già menzionato "Etna di sospiri, e un mar di pianto». ${ }^{39}$ In sintonia con ciò, Adriana, incaricando personalmente un autore posteriore a lei sconosciuto, desidera che venga trascritta la sua infelice storia d'amore come causa di «caldi sospir» e «pietose lacrime». ${ }^{4}$ La derivazione programmatica dell'effetto emotivo della "pietà" proprio da una storia d'amore tragica come lo è l'Adriana, trova un consolidamento teorico autorevole nelle argomentazioni di Sperone Speroni in difesa della sua Canace contro critici come Giraldi 
Cinthio. Su questo tema Speroni tiene un'intera Lezione all'Accademia degli Elevati a Padova: egli è fautore della tesi che dalle azioni degli innamorati possa, proprio a causa delle predisposizioni emotive del

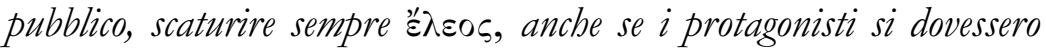
macchiare di "sceleratezza". ${ }^{41}$ Groto intende nella sua Adriana approfittare di questa opzione teorica, lasciando da parte la dimensione della "sceleratezza", in modo da ottenere, secondo la sensibilità del tempo,

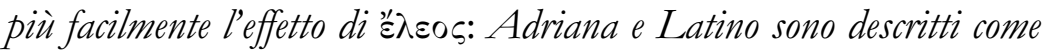
caratteri eticamente positivi; sono, se non altro, persone "mezzane", tendenti a una morale quasi integerrima. L'amore di Adriana come sentimento "sincero" si oppone orgoglioso (suscitando simpatia) alle pressioni e agli attacchi di ira dei genitori, senza pertanto nascondere il fatto che Latino in battaglia avesse ucciso il fratello di lei. Questa circostanza viene giustificata con l'argomentazione che il fratello è andato in battaglia in incognito, travestito e contro il volere del padre. Pertanto Latino non poteva identificare il suo nemico come fratello di Adriana. Cosi anche l'amante è libero da ogni sospetto di "sceleratezza" e la coppia di protagonisti attira su di sé tutta la simpatia. Il loro errore tragico e mortale, che segue il modello della storia di Piramo e Tisbe, ha chiaramente come obiettivo la compassione come effetto emotivo di base. La linea della tragedia dell'orrore viene esclusa nell'Adriana in maniera metapoetica nominando più volte Medea, normalmente un segnale per il modello drammatico dell'orrore "senecano", che qui al contrario non indica in senso metapoetico questa linea. ${ }^{42}$ Resti del repertorio orrorifico si trovano nelle reazioni irose dei genitori al rifuto di Adriana di unirsi, per ragioni dinastiche, in matrimonio con il giovane rampollo straniero: soprattutto il padre minaccia Adriana subito di morte e le prospetta di farsi vedere ancora più spietato di Eolo e di altre figure esemplari. ${ }^{43}$ E evidente il richiamo ai terrificanti eventi della Canace di Speroni, dove Eolo uccide o spinge ad uccidersi suo nipote, nato da un rapporto incestuoso, e i propri due figli. Ma la configurazione emotiva e situazionale dell'azione nell'Adriana non lascia spazio a un simile orrore; al contrario, la sfortuna dei protagonisti viene evocata involontariamente e risulta proprio dal tentativo di sfuggire a priori alla violenza paterna.

Neanche i riferimenti ai generi, che Groto introduce nell'Adriana per $i$ suoi tentativi di accostamenti vari di genere, precedentemente tracciati, permettono lo svilupparsi di una tragedia dell'orrore. La dizione 
petrarchista che ricopre tutto, la sua amplificazione senza limiti, tesa a creare un sottofondo di lacrime, lamenti d'amore ed esistenziali, porta all'erosione dell'insieme dell'azione tragica e al suo indebolimento rispetto all'ostentazione del linguaggio petrarchista: cosi si ha la strana impressione di una miscela tra l'azione che ritarda, l'unità di tempo che viene dilatata e l'unità di luogo che svanisce sotto una massa di parole manieriste. ${ }^{44}$ Cosi, al contempo, sotto un velo strappalacrime consacrato unicamente al pianto degli eventi d'amor tragico, vengono nascoste anche a prima vista le vere cause dei potenziali orrori. I riferimenti integrati nella tragedia a topoi provenienti dall'epica (come, ad esempio, $i$ dettagliati resoconti degli eventi bellici fuori dalle mura della città), ${ }^{45}$ oppure dal romanzo e dal poema eroico (come il travestimento del fratello di Adriana che cosi combatte con Latino e per colpa del quale sarà ucciso), ${ }^{46}$ conferiscono all'azione amorosa una nota romanzesca lontana dalla Stimmung della tradizione senecana.

L'indebolimento dell" intreccio"47 drammatico, al quale abbiamo già accennato, cosi come lo squilibrio nell'elaborazione dei caratteri dei genitori (prima attacchi di ira e minacce di morte alla figlia, poi coinvolgimento totale davanti alla sua morte, in primis soltanto supposta), ${ }^{48}$ sono due circostanze che banno soltanto la funzione di configurare la situazione amorosa sofferta di Adriana, collaborando a scardinare le norme poetiche in uso. ${ }^{49}$ Inizialmente l'effetto di compassione, che si vorrebbe ottenere, ha un gusto alquanto cartaceo, poiché $i$ personaggi non risolvono $i$ loro conflitti semplicemente in un mondo dell'azione autonomo, ma chiamano ripetutamente in causa per le loro azioni parallelismi letterari, e cosi anche qui la letterarietà e l'artificiosità del testo rimangono presenti. La balia, per esempio, tenta di distogliere la sua protetta Adriana dall'amore per Latino, ricordandole $i$ racconti mitologico-letterari di «Adrianna» (Ariadne: verso concettistico "Ricordati, Adriana, d'Adrianna», I.I v. 297), di Medea (cfr. supra), di Scilla e Ipsipile, ${ }^{\circ}$ mentre Latino, non sapendo ancora il destino che lo aspetta, paragona se stesso e Adriana a Orfeo e Euridice. $^{5 \mathrm{I}}$

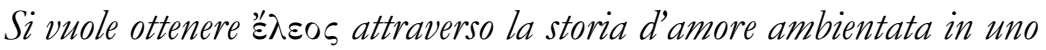
scenario tetro che conferisce un'ulteriore dimensione alla sofferenza dei protagonisti, soprattutto nel finale della tragedia. Questa sofferenza $\grave{e}$ inserita al centro di una visione del mondo complessivamente cupa. Qui non ci sono vere colpe e alla fine neanche una tragica á $\mu \alpha \rho \tau i \alpha$, inter- 
pretabile con parametri aristotelici, ma al livello dell'azione dei personaggi c'è semplicemente un fraintendimento oggettivo e temporale come causa della catastrofe letale: Latino riceve l'informazione sulle circostanze della morte soltanto apparente di Adriana nel momento in cui lui stesso ha già preso il veleno mortale; l'ultimo dialogo tra gli innamorati è segnato da una morte causata da un lapsus tecnico. L'importanza di questa circostanza viene sminuita dal finale, poiché la sofferenza personale rappresenta unicamente una minuta parte della configurazione tragica di Groto. La morte degli amanti è soltanto un piccolo riverbero della grande apocalisse causata dal padre di Latino e dalla sua vendetta gratuita motivata da una visione immateriale. ${ }^{52}$ Questi scatenando un'azione bellica inonderà tutta l'area di Adria (tutti i personaggi coinvolti nella trama della tragedia periranno pochi istanti dopo la chiusura del sipario nelle masse d'acqua che infuriano, ${ }^{33}$ e l'antica città di Adria sarà distrutta), ${ }^{54}$ e questa grande apocalisse a sua volta è solo un elemento dello scenario desolante di un'esistenza umana svuotata di senso. Solo quella cornice per cosi dire esistenzialista, in cui risuona il lamento d'amore dei protagonisti, conferisce loro importanza. ${ }^{55} \mathrm{La}$ gratuità e l'impenetrabilità della catastrofe e un ineluttabile "danno universales ${ }^{56}$ sono $i$ costituenti di una tale oscurità, nella quale la sciagura non ha mai fine. ${ }^{57}$

Se cosi sul tenebroso scenario dell'Adriana aleggia una paralizzante tristezza, nella Dalida domina il yóbos, nel senso del ribrezzo, dello shock e dell'orrore. Immediatamente, all'inizio del prologo, la tragedia viene esplicitamente delimitata rispetto agli altri registri di genere: chi si aspettasse di trovare sulla scena qualcosa di allegro o piacevole, cosi esordisce il testo, farebbe meglio ad abbandonare immediatamente la sala (un primo cenno dell'appartenza del testo alla tragedia d'orrore, in quanto anche all'inizio dell'Orbecche viene invitato lo spettatore sensibile - in previsione degli orrori che seguiranno - a lasciare il teatro). ${ }^{58}$ Non si assisterà a una commedia ("le argutie, e i motti / Di sal conditi da Sosia, ò da Siro»), ma neanche a un dramma pastorale («gli amor semplici, e vaghi / De le vezzose, e leggiadrette Ninfe, / E le rime cantate da Pastorì). ${ }^{59}$ Verrà piuttosto rappresentata una tragedia di «tenebroso horrore», di «miserie», fatta di «lamenti [...], lacrime, e mortì. ${ }^{6 \circ}$ Cosi Dalida è presentata come esibiz̨ione di $\pi \dot{\alpha} \theta \eta$ in senso aristotelico. ${ }^{61}$ Il rimando implicito del prologo di Groto all'Orbecche viene completato da un rimando esplicito al testo teatrale 
di Giraldi inteso come dramma fondativo di un (sotto)genere ("la Orbech [sic] modelo [sic] dell'altre»). ${ }^{62}$ Groto inserisce attentamente, tramite una serie di rimandi, la Dalida nella tradizione del genere tragico a partire dall'antichità, ${ }^{63}$ in seguito la connota come un elemento della falange delle più importanti tragedie classiciste del Cinquecento, ${ }^{64}$ e infine chiarisce - non solamente attraverso i rimandi al testo fondativo dell'Orbecche - la sua appartenenza al sottogenere delle tragedie d'orrore sulla linea Seneca-Giraldi. "5s A partire dal testo teatrale viene dichiarato, con chiarezza programmatica, che la Dalida è concepita come incremento massimale della "tragedia d'horrore". Berenice annuncia che il pasto orrendo da lei preparato, che verrà servito a Candaule, supererà di gran lunga in ribrezzo i tremendi menu cannibali della tradizione d'orrore: «Perche le mense / Di Tantalo, di Tereo, e di Thieste, / Rispetto à questa dispietata cena, / Possan quei, che verran, nomar pietose». ${ }^{66}$ Questo annuncio viene prontamente rispettato dal dramma. Qui le inserzioni d'obbligo di Groto, prese dal repertorio del petrarchismo lirico acuito manieristicamente, non modificano minimamente la violenza dell'orrore. Esse servono, piuttosto, a mettere in risalto $i$ rapporti tra $i$ protagonisti dell'azione, caratterizzati da una tensione affettiva, da un desiderio focoso e dai corrispondenti eccessi di gelosia e autoaffermazione. Candaule, ad esempio, invia a Dalida in un discorso concettistico uno specchio, in cui contemplare l'oggetto del suo desiderio (un motivo ricorrente nelle Rime di Groto); ${ }^{67}$ lo specchio viene poi però consegnato dal segretario, in una situazione formulata dettagliatamente secondo lo stile lirico-manierista, alla regina come taciuta dichiarazione d'amore; ${ }^{68}$ il segretario finge di fronte a Berenice un supposto corteggiamento in un alto registro petrarchista da parte di Candaule; ${ }^{69}$ quest'ultimo piange in una forzata dizione manierista-petrarchista la morte di Dalida trucidata. ${ }^{70}$ Viene aperto, in una sorta di effettivo sarcasmo metapoetico, un drastico contrasto tra il campo di validità del manierismo lirico e il mondo orribile della tragedia, quando il segretario riferisce del tutto erroneamente l'oracolo di Apollo «e man tronche, la lingua / Svelta, e tratti doveano esserti gli occhi> quale metafora petrarchista - da prendere invece alla letteraper la sua condizione di amore non corrisposto nei confronti della regina: ${ }^{7 \mathrm{I}}$ in questo cosmo tragico le mani, le lingue, gli occhi non vengono mozzate, strappate, cavati in maniera metaforica e allegorica, ma fisicamente. 
Groto nella Dalida scardina, a livello di genere poetologico, i teoremi e i postulati centrali dell' aristotelismo. ${ }^{72}$

(a) Non viene creato un arco di tensioni in senso tradizionale. Contro le pretese delle teorie a lui coeve, Groto non nasconde allo spettatore alcuna informazione, non gioca con una sproporzione delle informazioni al fine di creare suspense. ${ }^{73}$ Non c'è alcuna peripezia o agnizione al di là della rivelazione finale, che il pubblico fin dall'inizio si aspetta, tramite l'esibizione delle membra mutilate e delle teste delle vittime. L" "ombra" di Moleonte annuncia fin dall'inizio, con una chiara allusione, il pasto tiesteo che verrà servito a Candaule alla fine della tragedia, in modo tale che il pubblico dell'epoca non possa in alcun modo equivocare. ${ }^{74}$ Anche l'amore del segretario per Berenice e la gelosia della regina (che alla fine risulterà mortale), scaturita dalle rivelazioni del segretario, sono condivisi all'inizio della tragedia, in maniera inequivocabile, dall" "ombra" con lo spettatore, in vista degli atti successivi. ${ }^{75}$ La corrispondente informazione anticipata pone inoltre lo spettatore nella condizione di comprendere correttamente l'ironia tragica, forzata e carica di dolore, esposta in una serie di esternazioni dei protagonisti (e conformemente alla poetica dell'orrore, di cadere nel terrore). Quando Candaule cerca di formulare un'allegoria che miri ad esprimere il proprio affetto per la sua seconda famiglia («io non voglio cibo altro, che lei [Dalida], / Altro, che quelle delicate membra, / E que' mei dolci, e teneri fanciulli»), ${ }^{76}$ viene già evocato l'orribile pasto dell'ultimo atto. E quando egli, pianificando di uccidere Berenice con una corona di fiori preparata con una sostanza tossica, sottolinea la sicurezza di quel metodo, qualora Berenice non volesse utilizzare lo stesso contro di lui, allo spettatore diventa subito chiaro il terribile meccanismo di questa tragedia: sarà esattamente questo che accadrà. ${ }^{77}$ In Dalida è fin dall' inizio annunciato inequivocabilmente che accadranno cose orribili: l'azione, dal punto di vista dell'osservatore, non consiste in una curva ricca di tensioni costituita da agnizione e peripezia, bensi esige solo che si attenda l'avvento di un orrore già individuato.

(b) Una classificazione etica delle azioni dei protagonisti come buone / legittime o cattive / illegittime diviene complessa. La versione iniziale dell'antefatto dettagliatamente riportata (in I.I e I.2) dalla prospettiva dell" "ombra" di Moleonte (che dipinge Candaule come un usurpatore amorale e privo di scrupoli, Dalida come una meretrice perversa ed eroticamente deviata, e sembra rispondere in maniera chiara alla 
questione di chi sia colpevole) contrasta con una versione non chiarificata e più tarda, declamata a Berenice dal segretario, che fa apparire Moleonte come il tipo di tiranno avido di potere e privo di scrupoli, che ha voluto strappare di mano a Candaule il regno ereditato e che ha fatto gettare in carcere la madre, sua cognata (2.3). Qui ognuno è nel torto, $e$ questa tragedia non sembra indicare i propri protagonisti - come fa, tra le altre cose, la teoria del Cinquecento tratta dalla Poetica di Aristotele (cap. 2) - come persone moralmente "migliori", ma al contrario li presenta come mostri, ${ }^{78}$ di cui solo l'"esser migliori" a livello sociale, di fronte al pubblico del teatro, ne garantisce un punto a favore.

(c) L'agire dei protagonisti non è motivato in maniera chiara: da un lato i piani di vendetta come quello di Berenice vengono giustificati con l'ingresso dall'esterno di anti-virtù personificate come la Gelosia, ${ }^{79}$ che appunta al petto della regina una maligna pietra preziosa a lei corrispondente. Dall'altro lato però la vendetta di Berenice è già fin dall'inizio fondata sulla sua indignazione per le scoperte di Besso (2.3); non si tratta in alcun modo del dominio esclusivo di una forza esterna sull'agire umano, per cui qualcuno possa venir sollevato dalla responsabilità per le atrocità perpetrate. ${ }^{80}$ I protagonisti della Dalida operano secondo una scelta consapevole e si decidono per l'opzione del delitto. ${ }^{81}$ Questa "sceleratezza" cosciente, che conduce coloro che agiscono a una "colpa" intenzionalmente massimizzata, totalmente priva di freni e remore, ${ }^{82}$ impedisce che vi sia, tramite il rimando ai loro affetti, un certo qual alleggerimento che valga come scusa per il loro agire, come sarebbe stato possibile attendersi secondo la teoria della tragedia. ${ }^{83}$ La Dalida pone ad acta l'accesa discussione condotta tra Giraldi e Speroni, se lo spettatore possa partecipare o meno al dolore di "persone scelerate". ${ }^{84}$ La reazione primaria per $i$ crimini che avvengono sulla scena qui non è compassione, ma orrore e shock. Laddove tutt'al piu le vittime, in special modo i "fanciulli", possono risvegliare una certa "compassione", 85 sta di contro, in primo piano, la quasi perversa brutalità della loro liquidazione, che produce, come sentimento generale, yóbos.

(d) Il fatto che la "vendetta", sempre nuovamente tematizzata dalle figure principali, accada in maniera cosi smodata e drastica, potrebbe trovare la sua giustificazione, con un riferimento alla teoria del dramma cinquecentesco, nel tentativo di salvataggio di un nucleo di integrità etico: le azioni di vendetta del sovrano debbono avvenire in grande formato, per essere adeguate al suo rango. Questo stratagemma argomentativo 
viene addotto a livello teorico non solo dai contemporanei, ${ }^{86}$ ma impiegato anche dai protagonisti della tragedia. Candaule giustifica con questa argomentazione in maniera esplicita la sua «crudele, anzi giustissima vendetta». ${ }^{87} \mathrm{E}$ la sua - fino a quel momento - consorte (e futura assassina) Berenice farà esattamente la stessa cosa. ${ }^{88} \mathrm{Ma}$ nel testo teatrale stesso, l'unica figura positiva, il Consigliere del re, che combatte una battaglia persa in partenza, distrugge questa giustificazione. Candaule non ha alcun diritto, a causa di un'infrazione sociale, che egli vuole vedere nell'adulterio della regina, di intraprendere un'azione di vendetta di formato "regale". Egli e la regina avviano piuttosto una deleteria strategia dell" "occhio per occhio" e non tengono presente che: «ingiusto è lo sdegno di colui, / Che si sdegna patir quel, che già fece». ${ }^{89}$

Il Consigliere rappresenta però una posizione isolata. Malgrado egli rimandi ${ }^{\circ}$ ripetutamente, per il suo tradizionale concetto di giustizia, a modelli fondativi religioso-teologici, e malgrado il coro, scandalizzato dal resoconto della morte per avvelenamento di Berenice, faccia brevemente riferimento alla speranza ne "La giustitia di Dio santa, immortale», ${ }^{\mathrm{I}}$ qui il mondo dell'azione indicato è chiaramente abbandonato alla propria distruttività, e non va salvato dall'intervento divino. Anche altre figure, infatti, non banno alcuna fiducia in una qualche assistenza divina..$^{2}$ Il mondo della Dalida è tanto privo di speranza quanto quello dell'Adriana, e lo stesso Consigliere deve alfine riconoscerlo, quando dice a Candaule: "tosto andrete / Fuor di questo protervo immondo mondo, / Dove'l nascere è pena, / Il vivere è fatica, il morir forza, / Dove mai non si prova hora tranquilla, / Anzi il nostro babitar sopra la terra / E una continua guerra». ${ }^{93}$

Con cio il dittico di Groto si contrappone alla funzionalizzazione moralizzatrice-pacificatrice del genere tragico, ancora più radicalmente dell'Orbecche di Giraldi. Giraldi, come è noto, aveva pur sempre proposto una giustificazione moralizzante della sua tragedia d'orrore tramite una corrispondente interpretazione del meccanismo - presupposto ancora funzionante - della catarsi tragica. Groto, la cui Dalida riprende le strutture conflittuali basali dell'Orbecche (le mortifere fobie di perdita del potere e gli eccessi di gelosia che si rivolgono alla generazione dei figli operanti in segreto e ai loro figli) e li potenzia in un'azione annientatrice simmetricamente raddoppiata, radicalizza gli schemi di Giraldi tratteggiando a tinte ancor più fosche un cosmo 
contrassegnato ovunque dalla distruqione. Non solo perché entrambe le tragedie grotiane si concentrano ciascuna su un effetto emotivo aristotelico (invece di farlo su entrambi insieme, come la teoria e la prassi di Giraldi ufficialmente ancora tentava) non può qui più avvenire alcuna catarsi, questa infatti non viene neanche piu in alcun modo asserita da Groto, al contrario del tentativo di Giraldi ${ }^{94}$ di assicurare ancora alla tragedia uno scopo effettivo di tipo morale. L'effetto di apprendimento razionale di entrambe le sue tragedie può derivare solamente da una onnicomprensiva meditatio mortis, ${ }^{95}$ che si localizza lontano dall'ideologia della "vendetta" rappresentata dagli agenti nel mondo dell'azione. La conquista affettiva a partire da questa meditatio è una forma di '̇ं $\varepsilon_{0} s$, che va ben oltre una reazione all'agire dei personaggi in questa tragedia e oltre il sentimento per le (o in rapporto alle) figure tragiche. La loro motivazione costituisce il più tetro riconoscimento dell'inevitabilità dell" "esser-abbandonata" dell'esistenza umana. Nell'ambito delle forme umane di esistenza, la sventura, che costantemente si rinnova, conosce tutt'al più graduali smussamenti. Emerge cosi, come consolazione minima per l'individuo, (che anche l'autore Groto, secondo ciò che ci dice l'annunciatore del prologo, si augura per se stesso), lo sguardo gettato ai prossimi ancor più sofferenti. ${ }^{97}$

Il generale pessimismo che marca entrambe le tragedie di Groto ha fatto etichettare questi testi teatrali come manifestazioni di «angoscia manierista). ${ }^{8} \mathrm{Vi}$ sarebbe anche un accenno al fatto che il coinvolgimento della corte a tali drammi (un problema che a lungo termine Giraldi tentò di risolvere tramite la svolta dalle tragedie d'orrore alle tragedie con "lieto fine"), ${ }^{99}$ e soprattutto la loro collocazione in un discorso culturale socialmente stabilizzato, sono impossibili. Quando il Consigliere, nella Dalida, riconduce la maggior parte delle cause del devastante sviluppo dell' azione alla da lui condannata "adulazione" propria

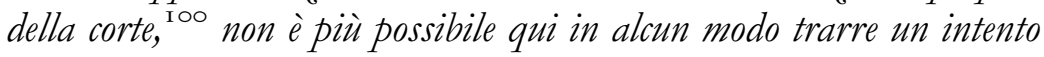
educativo dell'autore, che si rivolga ai deficit morali delle società cortesi; bensi gli adulatori di corte si mostrano molto di più come una forma visibile particolarmente potenziata di un mondo che ̀̀ abbietto nel suo complesso. Se Giraldi aveva tentato, con $i$ suoi pezzi successivi all'Orbecche, di "salvare" il mondo attraverso un'attenuazione della radicalità distruttiva della sua prima opera, e di mantenere le tragedie compatibili con l'ambiente di corte, ${ }^{\text {Iо I }}$ il letterato di provincia Groto, non legato all'ambiente di corte, rincara il radicalismo della visione 
tragica del mondo fino all'estremo. Tutti $i$ tentativi di scrivere una tragedia "ragionevole", formulati - ciascuno a modo proprio - nella Sofonisba di Trsissino, nell'Orazia di Aretino, nella Merope di Torelli, ${ }^{102}$ sono qui di gran lunga rifiutati. Groto mostra le estreme conseguenze che possono scaturire da una visione del mondo priva di salvezza del genere tragico. Questo è l'antipode più chiaro alla funzionalizzazione ${ }^{\mathrm{IO} 3}$ controriformista che si è voluta dare a questo genere nel Secondo Cinquecento.

Bernhard Huss (traduzione dal tedesco di Maraike Di Domenica) 
I. Un breve compendio dei più importanti contributi alla ricerca, complessivamente piuttosto esigua, viene offerto da Mott-Petavrakis I992, pp. I 3-22, che trascura però l'importante volume degli atti del congresso di Brunello-Lodo i 987 (vol. I).

2. Un compendio biografico e una breve descrizione delle opere di Groto vengono forniti nel conciso studio di Benvenuti del i984. Altri cenni biografici e informazioni sull'ambiente sociale di Groto si trovano in Rizzi 1987; cfr. anche Mott-Petavrakis I992, pp. 9-1 3 e Gallo 2003. La vecchia biografia di Bocchi del I 886 non è per questo del tutto obsoleta; tra le altre cose fornisce un elenco delle opere particolarmente utile per la storia editoriale dei testi di Groto tra il i 586 I 886 (pp. 93-104).

\section{Mott-Petavrakis i 992, p. 9.}

4. Cfr. Huss $2014 \mathrm{a}$ e 20I4b con ulteriori indicazioni bibliografiche.

5. La maggior parte dei testi teatrali è stata rappresentata, anche se non frequentemente; per quanto riguarda le date delle rappresentazioni cfr. Zampolli $200 \mathrm{I}$, p. 94 note 4- I ; Spaggiari 2009, p. I 89s. nota I 2; inoltre, in particolare rispetto alle due tragedie Pieri 1979 , p. 23 nota 39.

6. Cfr. oltre all'edizione dell'Adriana con introduzione e commento di Ariani (Luigi Groto, Adriana) l'importante capitolo riguardo l'Adriana come caso esemplare di una tragedia manierista in Ariani 1974a, pp. I79-230, qui pp. 2 I 2-30. Altre considerazioni sull'Adriana, soprattutto riguardo al suo petrarchismo stilistico, in Huss $201 \mathrm{I}$, pp. 240-44.

7. Soltanto come esempio rispetto alla questione vedi Cavazzini 1987 e Bazoli 2004 .

8. Per la storia editoriale iniziale dei drammi di Groto cfr. il riassunto in Zampolli 200I, p. IO3s.

9. Ariani I977, p. 282 e p. 284 elenca per l'Adriana complessivamente Io edizioni fino al I626; così anche Pieri I979, p. 23 nota 39, che inoltre elenca per la Dalida complessivamente 8 edizioni fino al $\mathbf{6} 646$.

ı. Cfr. in dettaglio Huss $2014 \mathrm{~b}$.

I I. Cfr. a questo proposito Pieri i979, p. 5 con nota 8.

I 2. Cfr. in maniera circostanziata Huss $2014 \mathrm{a}$ e, specificamente rispetto al contesto della drammatica di Groto, Zampolli 2000, pp. 37-42.

I3. Ciò succede in maniera sistematica nelle lettere di Groto proprio dal momento della stesura della Dalida, come dimostra Huss 20 i 4a. Dal i 57 I Groto usa indicare nella sua corrispondenza come mittente «Luigi Groto cieco d'Adria».

I4. In Adriana la patetica storia d'amore secondo lo schema Romeo-Giulietta (ripreso dalle novelle di Luigi da Porto e Bandello) viene ambientata nella città 
antica di Adria. L'amore infausto tra i giovani Adriana e Latino, appartenenti a due casate nemiche e in guerra tra loro, finisce con la morte di Latino che, alla tomba della presunta morta, in verità soltanto intenzionalmente narcotizzata, si avvelena. Adriana a sua volta, svegliatasi poco prima della morte dell'amato Latino, si dà la morte con un pugnale. La città di Adria invece sarà distrutta da un'inondazione causata intenzionalmente dal padre di Latino.

I 5 . Nella Dalida il sovrano battriano Moleonte, spodestato e ucciso da suo nipote Candaule, prende le sembianze della sua "ombra" e causa con l'aiuto delle personificazioni di Gelosia e Morte una duplice distruzione, motivata dalla sete di vendetta: il re Candaule aveva precedentemente sposato Berenice, la figlia del re indiano, e si era congiunto in seguito segretamente con Dalida, la figlia di Moleonte, da cui ebbe due figli. Besso, segretario di Candaule, rivelerà questo rapporto adultero a Berenice e verrà ricompensato da lei per il suo seguente servigio con l'atto sessuale da lui tanto agognato. In seguito Besso verrà ucciso $\mathrm{e}$ fatto a pezzi dallo stesso Candaule. Quasi contemporaneamente Dalida e i suoi figli saranno uccisi e smembrati da una Berenice depravata a causa della gelosia e del sadismo. Candaule e Berenice si presenteranno a vicenda i resti di questi atti orribili (Candaule ha già consumato i resti dei suoi cari, simile alla cena di Tieste) rivelandosi di aver avvelenato l'un l'altra, e infine moriranno di una atroce morte per veleno.

I6. Groto, Adriana, p. 289 (Prologo, vv. 78-90): «La cui istoria, scritta in duri marmi / (ma men duri però de la lor fede) / trovò l'autor, con queste note chiusa: / "A te, che troverai dopo tanti anni / la scoltura di questo acerbo caso, / si commette, che tu debbi disporlo / in guisa che rappresentar si possa, / porgendo un vivo esempio, in quella etate, / d'un amor fido, ai giovani e a le donne, / benché piú lungo spazio ti convenga / stringer di tempo che non porta l'uso. / Del che, per iscusarti, hai qui licenza / d'aggiungere una parte anzi il principio."»

17. Groto, Adriana, p. 4 I 8 (5.8, vv. 59-69); cfr. p. 420 (5.8, vv. I I4-і I 7), dove il "Mago" assicura, che indirizzerà, come richiesto, l'incarico della scrittura al futuro e ancor sconosciuto autore. Per questa metalessi cfr. Zampolli 2006, p. 7 os., p. 73 s.

I 8. Cfr. Groto, Adriana, p. 287 (Prologo, vv. I7-47) e pp. 420-24 (5.9 passim [scena finale]).

19. Anche qui troviamo un'apposita giustificazione del Prologo, che generalmente è elemento tipico della commedia; cfr. Luigi Groto, La Dalida, p. I4.

20. Già all'inizio del Prologo dell'Adriana (p. 287, vv. 7-I I) troviamo un indizio simile, ma meno esteso: La cecità empirica dell'autore («l'autor [...] / pien d'ogni oscuro, e tragico accidente, / che chiusi avendo in nube eterna gli occhi») è una spiegazione, per la sua natura dolorosa, per il fatto che non soltanto chi recepisce la tragedia commovente dell'Adriana venga indotto a piangere, ma anche, che proprio questo autore, che versa tante lacrime lui stesso, abbia scritto un dramma di questo tipo; cfr. Zampolli 2006, p. 72 nota 29. 
2 I. Tutte le citazioni precedenti da Groto, La Dalida, p. i I.

22. L'espressione è di Pieri I979, p. I 8 (cfr. il contesto dell'argomentazione).

23. Groto, La Dalida, p. i .

24. Groto, La Dalida, p. I 2.

25. Groto, La Dalida, p. I 2s.

26. Groto, La Dalida, p. I 3.

27. Groto, La Dalida, p. I 3.

28. Mentre le sofferenze della figura auctoris nelle Rime vengono correlate ai "danni d'amore" petrarchisti (Huss 2014a, p. 79s.), nella Dalida queste stesse sofferenze vengono messe in relazione attraverso una diversificazione conforme ai generi con i tragici $\pi \dot{\alpha} \theta \eta$.

29. Groto, La Dalida, p. Ioo (3.6).

30. Groto, La Dalida, p. 8 is. (3.I).

3 г. Cfr. Pieri i 979, p. i6s. e il contesto argomentativo.

32. A tal proposito cfr. Ariani i 979, p. i I7.

33. Pieri 1979, p. 17, che non vuole sottolineare gli opposti "compassione" e "orrore", ma l'opposizione tra la tragedia senecana e la tragedia d'amore patetica (p. I7s.). Implicitamente anche Herrick 1965 ha intuito l'opposizione tra i due drammi e ha considerato l'Adriana una delle "Gothic and Romantic Tragedies», tragedia sentimentale e patetica, mentre la Dalida viene da lui trattata nel capitolo «More Blood» (quindi tra le tragedie dell'orrore sulla scia dell'Orbecche).

34. Così per es. in Nicolò Rossi, Discorsi intorno alla tragedia, p. I I $7 \mathrm{~s}$.

35. Rispetto al distacco di Groto dai parametri fondamentali della norma classicista cfr. Zampolli 2000 , pp. 30-37.

36. A questo proposito cfr. essenzialmente Di Domenica 20I4. Rispetto al ruolo pionieristico di Giraldi in questo contesto cfr. Ariani 1971; Ariani 1974b; Ariani I 979 .

37. Cfr. Pieri i 989 , p. I 37 s.

38. Notabene: questi sottogeneri sono definibili soltanto rispetto ad una graduazione tipologica, cioè la predominanza della compassione rispetto all'orrore o viceversa, non nel senso di una esclusione reciproca dei due effetti emotivi.

39. Groto, Adriana, p. 287 (Prologo, v. 2 e 6). 


\section{Bernhard Huss}

40. Groto, Adriana, p. 4i 8 (5.8, v. 66s.). Sull'Adriana come tragedia d'amore nel senso di una tragedia "strappalacrime" cfr. anche Huss 20 I I, p. $240 s$.

4i. Sperone Speroni-Giambattista Giraldi Cinzio, Canace e Scritti in sua difesa. Scritti contro la Canace. Giudizio ed Epistola latina, pp. 220-34.

42. In i.I, vv. 307-3 I 3 (Groto, Adriana, p. 302) la balia, che vuole distogliere Adriana dal suo amore, porta come esempio deterrente di un'innamorata infelice proprio Medea (ma non come esempio di una infanticida). In 4.4, vv. I4-I 8 (Groto, Adriana, p. 389) Orontea, la madre di Adriana, si lamenta prima del funerale della figlia supposta morta (in verità in quel momento soltanto anestetizzata intenzionalmente): "Qual sarà quell'Oreste, / quell'Atreo, quel Tieste, / qual sarà quella rea, / quella Progne, o Medea, / che mi divida dal mio amato seme?» Questa sfilza di elementi tipici della tragedia dell'orrore subisce una frattura ironica; non ha luogo nessuna azione corrispondente a questo sottogenere e fino a quel momento non c'è stato neanche un caso di morte, e il pubblico, al contrario di Orontea, ne è ben a conoscenza.

43. Groto, Adriana, p. 36i (3.2, v. iо5).

44. Cfr. Huss $201 \mathrm{I}$, p. 243s. Rispetto alla questione dell'estensione dell'unità di tempo, già accennata nel Prologo dell'Adriana (cfr. supra), cfr. anche Ebner I 898, p. 137 e Bazoli 2004, p. 23 s.

45. Cfr. Groto, Adriana, pp. 306-I4 (1.2).

46. Groto, Adriana, pp. 3 I 4-23 (I.3).

47. Cfr. anche Ariani i 974a, p. 204.

48. Cfr. tra gli altri Groto, Adriana, pp. 35 2-56 (3.I) e pp. 358-6 I (3.2) vs. p. 383 (4.I).

49. Cfr. anche Ariani i977, p. XLVI, LIs.; Cavazzini I987, p. 347.

50. Groto, Adriana, p. 30 is. (I.I, vv. 297-325).

5 1. Groto, Adriana, p. 347s. (2.3, vv. 270-83).

52. Cfr. Groto, Adriana, p. 42 I (5.9, vv. 29-33).

53. Il rombo sordo di queste masse d'acqua è già udibile quando l'azione scenica sta per terminare: «Udite già il rumor che a noi s'appressa, / qual di molte moline accolto suono, / o come di celeste orribil tuono» (Groto, Adriana, p. 423 [5.9, vv. $103-105])$.

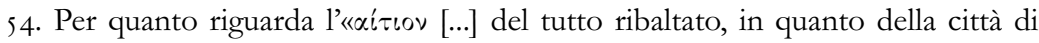
Adria non si canta l'origine e l'edificazione, ma la sua distruzione», e la fissazione per la catastrofe che ne consegue cfr. Ariani i 977, p. L; in questo contesto e per quanto riguarda l'estensione della tematica della sofferenza dai protagonisti alla collettività cfr. Zampolli 2006, p. 75 . 
55. Cavazzini 1987 , p. 345 sottolinea in questo contesto soprattutto il ruolo del paesaggio desolato, svuotato di senso e cupo premonitore di catastrofi, che viene evocato dal testo come scena dell'azione.

56. Cfr. Groto, Adriana, p. 423 (5.9, vv. 90-93): «Non lacrimate, donne, il vostro male, / tutta piangete a un tempo la cittate, / ché 'n danno universale / si disdicon le lacrime private.»

57. Le parole finali della tragedia sono: «Sol mai non giunge un mal, giungono molti, / sempre in drapel raccolti. / Per poco mai fortuna non comincia / a perseguire un misero. Ella il preme, / e mentre ei piange, in tanto / gli apparecchia cagion di novo pianto» (Groto, Adriana, p. 424 [5.9, vv. I 27-32]).

58. Giovan Battista Giraldi Cinzio, Orbecche, p. 85s. (Prologo, vv. 25-36). Peraltro il prologo giraldiano tematizza, malgrado la poetica dell'orrore che verrà installata tramite il suo dramma come linea dominante in Italia, non soltanto l'effetto emotivo di yóbos, bensì in particolar modo proprio anche l'effetto di

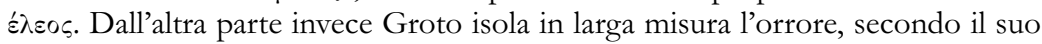
esperimento qui analizzato. Anche laddove gli orrori vengono comunicati o raffigurati, viene fatto in questa tragedia in maniera talmente forzata, che alla pietà per la vittima innocente si sovrappone a forza l'orrore di fronte agli agghiaccianti dettagli.

59. Groto, La Dalida, p. Iо.

6o. Groto, La Dalida, p. i I.

6i. Cfr. Giovan Battista Giraldi Cinzio, Discorso over lettera [...] intorno al comporre delle comedie et delle tragedie, p. 268 , dove vengono discussi come esempio di $\pi \dot{\alpha} \theta \eta$ «morti», «tormenti» $\mathrm{e}$ «ferite». Che Giraldi in maniera aristotelica annoti in proposito che questo conduca in egual misura «all'horrore et alla compassione» dimostra la distanza dell'esperimento di Groto nei confronti dell'aristotelismo coevo.

\section{Groto, La Dalida, p. 8.}

63. Candaule richiama, nei suoi rimproveri contro Berenice colpevole di adulterio con il segretario, l'esempio funesto «D'Egisto, e Clitennestra», quindi l'Agamennone di Eschilo (Groto, La Dalida, p. Io8 [3.6]). Anche Berenice nel suo piano vendicativo si ricollega all'esempio di Clitennestra (Groto, La Dalida, p. 68s. $[2.5])$.

64. La prefazione tematizza un riferimento poetologico «alle Rosimonde, alle Canaci, \& alle Didoni» (Groto, La Dalida, p. 4s.) e rimanda infine accanto all'Orbecche anche alla Gismonda (di Girolamo Rizzi; cfr. in proposito Herrick i 965 , pp. I 88-i 9o, che l'analizza come tragedie dell'orrore), alla Medea (ci si riferisce qui alla tragedia di Maffeo Galladei pubblicata nel i 558 ), alla Cleopatra (ci si riferisce qui alla tragedia di Alessandro Spinello pubblicata nel is50) come anche alla Sofonisba di Trissino (Groto, La Dalida, p. 8).

65. L"'ombra" di Moleonte instaura già in I.I un richiamo al caro soggetto tragico dell'orrore della storia di Progne (Groto, La Dalida, p. I 8) e aggiunge in 
I.2 un rimando all'uccisione del nipote di Eolo, quindi alla Canace (Groto, La Dalida, p. 2I). Durante il brutale resoconto fatto dal messo dell'uccisione di Dalida e dei suoi figli, l'“ombra" glorifica il racconto come appropriato «à far tragedie al mondo» (Groto, La Dalida, p. I 38 [4.2]). Berenice confronta il suo piano di vendetta con quello (dal momento fittizio dell'azione tematizzato proletticamente) dell'«animosa, e terribil Rosimonda» (Groto, La Dalida, p. 69 [2.5]).

66. Groto, La Dalida, p. I43s. (p. I44: errore di impaginazione «I46» [4.3]). Si noti inoltre qui l'impiego polivalente e raffinato di «pietose»: tramite l'orrore del nuovo banchetto tiesteo la "pietà" viene distanziata in nuova maniera.

67. Groto, La Dalida, p. 33 (2.1).

68. Groto, La Dalida, p. 62 (2.4).

69. Groto, La Dalida, p. 48 (2.3).

70. Groto, La Dalida, p. I 59 (5.2).

7i. Groto, La Dalida, p. 34 (2.2). Besso si riferisce con le «man tronche» alla propria impotenza, con la «lingua svelta» all'impossibilità di esternare il suo amore socialmente sproporzionato, con $\mathrm{i}$ «tratti $[\ldots]$ occhi» alla cecità del suo irrazionale desiderio d'amore.

72. Cfr. per le tendenze analoghe dell'Adriana ciò che è stato riportato sopra e ancora Ariani i974a, p. 215.

73. Per le teorie aristoteliche cfr. le argomentazioni di Nicolò Rossi sulla $\lambda u ́ \sigma \iota \varsigma$ tragica con l'esempio della Sofonisba: «questo è detto scioglimento perché slega e scioglie la favola, e fa palese quell'essito infelice della persona principale che sino allora era stato molto intricato et occulto» (Rossi, Discorsi intorno alla tragedia, p. I I 5 ).

74. Cfr. ad esempio in vocazione dell'assente Dalida: «Hoggi gli la [al tuo marito] darai de le tue membra [...] Dapoi, che tu questa passata notte / Con supremo desio chiedevi al cielo / Non ti disgiunger dal tuo sposo mai; / Io cura havrò, che questo don riceva, / E le membra con lui congiunga in modo, / Che nel suo corpo stia, nè mai te n'esca.» E subito dopo "l'ombra" mette in chiaro che i figli di Candaule non dovranno sopravvivere: «Bacia i figli, Candaule, mentre hai tempo, / Che non li bacierai più forse vivi. / Tu le figliuole sai privar di padre, / Edaltri [sic] il padre sa privar di figli» (Groto, La Dalida, p. 24 [1.2]).

75. Groto, La Dalida, p. 27s. (г.3).

76. Groto, La Dalida, p. 33 (2.I). Altri esempi di ironia tragica si possono accumulare come segue: lo spettatore conosce i piani di Berenice e rabbrividisce quando Dalida si reca in sua balia con le parole «E me medesma, e questi parti mei / Dono liberamente in poter vostro. / Voi ne potete far ciò che vi piace» (Groto, La Dalida, p. 9I [3.4]). Che Berenice non sia in nessun caso la madre di Candaule, come invece crede Dalida, è un fatto altrettanto noto al pubblico. 
77. Cfr. Groto, La Dalida, p. i I7 (3.8) con p. I43 (4.3).

78. Con ciò Groto si contrappone completamente ad un teorico come Nicolò Rossi, che annuncia: «benché le tragedie possano acontenere i costumi buoni et $\mathrm{i}$ cattivi, nientedimeno più eccellenti sono quelle che contengono i buoni solamente» (Rossi, Discorsi intorno alla tragedia, p. 97). Anche i tentativi di giustificazione di Giraldi del tipo tragico da lui inaugurato tramite un rimando ad una dura "punizione" con un fine educativo del protagonista "mezzano" (Giraldi Cinzio, Discorso, p. 23os.), vengono erosi da Groto con la messinscena di mostruosi "scelerati".

79. Groto, La Dalida, p. 58 (2.4), Berenice commenta ciò in maniera manierista con le parole: «Par che gran gelo sia [!] / Dentro al mio petto sparso».

80. Berenice diviene nel suo furor di gelosia una rappresentante del tipo cinquecentesco di protagoniste femminili demoniache; cfr. in generale Di Domenica 2014, p. I06 nota 46.

8 I. In questo modo Berenice in un monologo si infervora da sola: «Tu, Berenice, ogni gran prova ardisci, / Nè scelerata impresa ti spaventi» (Groto, La Dalida, p. 70 [2.5]), e dopo il massacro di Dalida e dei suoi figli, Berenice replica il disappunto del coro («Cotesta sceleraggine è pur grande») a sangue freddo solo con un: «Grande. Ma il duol maggior comanda farla» (Groto, La Dalida, p. I45 $[4 \cdot 3])$.

82. Anche qui la Dalida rappresenta un forte contrasto ad una teoria tragica, delimitata razionalmente, come quella del Rossi, che sotto il richiamo della "giudiziosa" Sofonisba postula un limite al volume della colpa tragica (Rossi, Discorsi intorno alla tragedia, p. 77).

83. Cfr. Rossi, Discorsi intorno alla tragedia, p. 75, che rimanda al forte effetto delle figure tragiche (come della Medea, della Canace o della Semiramis di Muzio Manfredi), che giustifica le azioni orribili, ma che non si esprime affatto riguardo all'babitus caratteriale e irreprensibile delle figure.

84. Cfr. Speroni-Giraldi Cinzio, Canace e Scritti in sua difesa. Scritti contro la Canace. Giudizio ed Epistola latina, p. 98, p. го I, pp. 228-34.

85. Questa potenza d'effetto dell'uccisione di bambini innocenti viene riflettuta espressamente da Giraldi nella sua polemica contro la Canace di Speroni; cfr. Speroni-Giraldi Cinzio, Canace e Scritti in sua difesa. Scritti contro la Canace. Giudizio ed Epistola latina, p. i 25. Per il ruolo dei "fanciulli" come vittime nella tragedia cinquecentesca cfr. in generale Neri I904, p. I44s. nota 4.

86. Rossi, Discorsi intorno alla tragedia, p. 76 : «E se sono reputate grandissime crudeltà quelle che usarono alcuni de' su detti personaggi, aviene ciò perché le persone eccellenti e poste in alto stato non si contentano delle picciole vendette, quando sono così gravemente offese». 


\section{Bernhard Huss}

87. Groto, La Dalida, p. Iо2 (3.6), cfr. ibid. p. io is. il contesto dell'intera replica di Candaule. Candaule si attiene espressamente a questa posizione anche in 3.8 (Groto, La Dalida, p. i I 3s.).

88. Groto, La Dalida, p. I42s. (4.3).

89. Groto, La Dalida, p. iоs (3.6).

90. Groto, La Dalida, pp. 74-78 (3.I), p. 95 (3.5).

9i. Groto, La Dalida, p. i69 (5.3).

92. In conformità a ciò il coro prega solo brevemente alla fine del primo atto: «Oे Giove, alto, immortale, / Ò leva in tutto, ò scema in parte il male» (Groto, La Dalida, p. 30).

93. Groto, La Dalida, p. i6 i (5.2).

94. Cfr. tra gli altri Giraldi Cinzio, Discorso, p. 2 I 3, p. 230 ., p. 3 I 6.

95. Proprio ciò deduce il coro nella scena finale dagli avvenimenti della tragedia (Groto, La Dalida, p. i 7 is. [5.3]).

96. Riguardo a questa cfr. il postulato dell'“ombra” di Moleonte: «Dalida, credi pur, sappi pur certo, / Che giunta con la colpa andrà la pena» (Groto, La Dalida, p. $23[\mathrm{I} .2])$.

97. Cfr. di nuovo Groto, La Dalida, p. I 3. Curiosamente Kindermann i 959, p. 67 ritiene ciò il messaggio per eccellenza della tragedia d'orrore rinascimentale, e precisamente all'insegna del cortese: «Die abenteuerlich-grausamen Züge des eigenen Renaissance-Zeitalters erscheinen so vor dem Spiegel noch viel entsetzlicherer Schicksale erträglich und fast selbstverständlich. Das Theater wird derart zum fürstlichen Trost: es gibt viel Schlimmeres als ihr hier mitmacht».

98. Cfr. Ariani i97 I, p. 448 per l'«angoscia manierista» dell'Orbecche. Anche l'analisi di Di Domenica 2014 si avvicina ad una prospettiva della tragedia d'orrore sotto il segno di un "anguished mannerism", cfr. ivi tra le altre p. i Ios. Sulla legittimità di tali considerazioni sulla "tragedia d'orrore" nemmeno lo scetticismo basilare riguardo alla portata generale del termine "anguished mannerism" può modificare qualcosa, come dimostra Regn 2014, p. 23 s. Cavazzini i 987, p. 349 s. nota 3, non utilizza il predicato "anguished mannerism", tuttavia classifica Groto come «organico esponente della crisi culturale del Cinquecento, crisi di profonda erosione degli istituti e delle pratiche letterarie rinascimentali». Cfr. anche d'Amelj Melodia 2009, pp. 6i 8-2I.

99. Per il problema di una compatibilità della tragedia con la corte, con focus su Giraldi, cfr. Ariani 1979, soprattutto pp. i 19-28.

ıоo. Cfr. Groto, La Dalida, pp. 92-96 (3.5). 
ıо . Cfr. a riguardo in modo esemplare l'analisi di Ariani 1974, pp. i65-76 (sulla Cleopatra di Giraldi).

I02. Cfr. a proposito ad esempio Pieri I989, tra le molte altre p. I37, p. I 54 ; per l'opposizione radicale delle tragedie d'orrore contro l'«equilibrio fiducioso» di Trissino cfr. anche Ariani i97I, p. 445.

I03. Per questo merita una precisazione l'affermazione di Doglio, secondo la quale Groto sarebbe diventato solamente per i suoi «temi novellistici» un opponente alla «tematica cattolica», che stava lentamente prendendo il sopravvento (Doglio ig6o, p. LXIV).

\section{BIBLIOGRAFIA}

Giovan Battista Giraldi Cinzio, Orbecche, in Il teatro italiano II, La tragedia del Cinquecento I, a c. di M. Ariani, Torino, Einaudi, I977, pp. 79-1 84.

Giovan BatTista Giraldi Cinzio, Discorso over lettera [...] intorno al comporre delle comedie et delle tragedie, in Discorsi intorno al comporre, rivisti dall'autore nell'esemplare ferrarese Cl. I 90, a c. di S. Villari, Messina, Centro Interdipartimentale di Studi Umanistici, 2002, pp. 205-318.

Luigi Groto, Adriana, in Il teatro italiano II, La tragedia del Cinquecento I, a c. di M. Ariani, Torino, Einaudi, I 977, pp. 28 I-424.

Luigi Groto, La Dalida. Tragedia nova, Venetia i 572 (Domenico e Giovanni Battista Guerra).

Nicolò Rossi, Discorsi intorno alla tragedia, in Trattati di poetica e retorica del Cinquecento 4, a c. di B. Weinberg, Bari-Roma, Laterza, I974, pp. 59-I 20.

Sperone Speroni-Giambattista Giraldi Cinzio, Canace e Scritti in sua difesa. Scritti contro la Canace. Giudizio ed Epistola latina, a c. di Ch. Roaf, Bologna, Commissione per i Testi di Lingua, I 982 (Collezione di Opere Inedite o Rare, I 38 ).

M. Ariani, L'Orbecche di G.B. Giraldi e la poetica dell'orrore, in «La Rassegna della Letteratura Italiana», 75 (197I), pp. 43 2-50.

M. Ariani, Il Manierismo e la dissoluzione della struttura tragica, in Tra classicismo e manierismo. Il teatro tragico del Cinquecento, Firenze, Olschki, 1974 (Accademia Toscana di Scienze e Lettere "La Colombaria", Studi 31), pp. 179-230 = 1974a.

M. Ariani, Ragione e furore nella tragedia di G. B. Giraldi Cinthio, in Tra classicismo e manierismo. Il teatro tragico del Cinquecento cit., pp. i 5-78 = 1974b.

M. Ariani, Introduzione, in Il teatro italiano II, La tragedia del Cinquecento I, a c. di M. Ariani, Torino, Einaudi i 977, pp. VII-LXXX.

M. Ariani, La trasgressione e l'ordine. L'Orbecche di G. B. Giraldi Cinthio e la fondazione del linguaggio tragico cinquecentesco, in «La Rassegna della Letteratura Italiana», 83 (i 979), pp. I I 7-80. 
G. Bazoli, Groto e Shakespeare: un confronto possibile?, in «Quaderni Veneti», 39 (2004), pp. 7-27.

G. Benvenuti, Il Cieco di Adria. Vita ed opere di Luigi Groto, Sala Bolognese, Forni, I 984 .

F. Bocchi, Luigi Groto (Il Cieco d'Adria), nato 8 settembre I54I - morto I3 dicembre 1585 . Il suo tempo, la sua vita e le sue opere, Adria, Eredi Guarnieri, I 886.

Luigi Groto e il suo tempo (I54I-I585), vol. I: Atti del convegno di studi, Adria, 27-29 aprile 1984, a c. di G. Brunello-A. Lodo, Rovigo, Minelliana, I987.

G. Cavazzini, Dall'Adriana a Romeo and Juliet: problemi di un rapporto, in Luigi Groto e il suo tempo (I54I-I585) cit., pp. 337-53.

V. d'Amelj Melodia, Atti e fatti nel prologo dell'Hadriana del Groto e della Marianna del Dolce, in Sacro e / o profano nel teatro fra Rinascimento ed Età dei lumi. Atti del Convegno di Studi, Bari, 7-Io febbraio 2007, a c. di S. Castellaneta-F. S. Minervini, Bari, Cacucci, 2009, pp. 6i 5-22.

M. Di Domenica, Manierismus vs. Aristotelismus. Zur ästhetischen Subversion regulativer Prinzipien in den Horror-Tragödien der italienischen Renaissance, in Manierismus. Interdisziplinäre Studien zu einem ästhetischen Stiltyp zwischen formalem Experiment und bistorischer Signifikanz, a c. di B. Huss-Ch. Wehr, Heidelberg, Winter, 2014 (GRMBeiheft 56), pp. 93-I I I.

F. Doglio, Il teatro tragico italiano. Storia e testi del teatro tragico in Italia, Bologna, Mareggiani, i 960.

J. Ebner, Beitrag zu einer Geschichte der dramatischen Einheiten in Italien, ErlangenLeipzig, Deichert, I 898 (Münchener Beiträge zur Romanischen und Englischen Philologie, I 5).

V. Gallo, Groto (Grotto), Luigi (detto il Cieco d'Adria), in Dizionario Biografico degli Italiani, vol. 6o, a c. di F. P. Casavola et al., Roma, Istituto della Enciclopedia italiana, 2003 , pp. 2 I-24.

M. T. Herrick, Italian tragedy in the Renaissance, Urbana, The University of Illinois Press, 1965 .

B. Huss, Petrarkismus und Tragödie, in Der Petrarkismus - ein europäischer Gründungsmythos, a c. di B. Huss-M. Bernsen, Göttingen, V\&R unipress, 20 I (Gründungsmythen Europas in Literatur, Musik und Kunst, 4), pp. $225-57$.

B. Huss, Figura auctoris und Selbstreferenz des poetischen Diskurses bei Luigi Groto, in «Germanisch-Romanische Monatsschrift», 64.4 (2014), pp. 407-427. Versione italiana pubblicata negli atti del convegno «IV Col.loqui internacional Mimesi: Metaficció - Renaixement \& Barroc», a c. di J. Solervicens / A. Lluís Moll, Universitat de Barcelona, 3.-4. Ottobre $20 \mathrm{I}_{3}=20 \mathrm{I} 4 \mathrm{a}$.

B. Huss, Luigi Grotos Rime: Manierismen als implizite Metapoesie, in Manierismus. Interdisziplinäre Studien zu einem ästhetischen Stiltyp zwischen formalem Experiment und bistorischer Signifikeanz cit., pp. 71-92 = 2014b. 
H. Kindermann, Theatergeschichte Europas 2: Das Theater der Renaissance, Salzburg, Müller, I959.

A. Mott-Petavrakis, Studien zum lyrischen Werk Luigi Grotos. Interpretation und literarbistorische Einordnung seiner Rime, Hamburg, Romanisches Seminar der Universität Hamburg, 1992 (Hamburger Romanistische Dissertationen, 23).

F. Neri, La tragedia italiana del Cinquecento, Firenze, Galletti e Cocci, I904.

M. Pieri, Il "laboratorio" provinciale di Luigi Groto, in «Rivista italiana di drammaturgia», 4 (1979), pp. 3-35.

M. Pieri, La nascita del teatro moderno in Italia tra XV e XVI secolo, Torino, Bollati Boringhieri, 1989 .

G. Regn, Manierismus: Kritik eines Stilbegriffs, in Manierismus. Interdisziplinäre Studien zu einem ästhetischen Stiltyp zwischen formalem Experiment und historischer Signifikanz cit., pp. I9-44.

F. Rizzi, Le socialità profonde: La famiglia di Luigi Groto Il Cieco d'Adria, in Luigi Groto e il suo tempo (I54I-I585), vol. I: Atti del convegno di studi, Adria, $27-29$ aprile I984 cit., pp. $23-60$.

B. Spaggiari, La presenza di Luigi Groto in Shakespeare e negli autori elisabettiani, in «Italique», I 2 (2009), pp. 173-198.

L. Zampolli, La réflexion théâtrale de Luigi Groto: de la critique des codes à l'autoreprésentation, in Le théatre réfléchi. Poétiques théâtrales italiennes des Intronati à Pasolini, a c. di F. Decroisette, Saint-Denis, Presses Universitaires de Vincennes, 2000, pp. 29-49.

L. Zampolli, "Una scena di perpetua durevolezza»: le projet théatral de Luigi Groto, l'avengle d'Hadria, in Théatre de cour, théâtre de ville, théatre de rue. Actes du Colloque International, 26-27-28 novembre 1998, a c. di R. Horville-O. Kleiman-G. Logez, Lille, Université de Lille 3, 200I, pp. 93-104.

L. Zampolli, Les voyages du témoin: le "destinataire privilégie" de L'istoria novellamente ritrovata di due nobili amanti (IS24) di Luigi Da Porto à La Adriana di Luigi Groto (I578), in Les traces du spectateur. Italie, XVII et XVIII siècles, a c. di F. Decroisette, Saint-Denis, Presses Universitaires de Vincennes, 2006, pp. $63-82$.

* Una versione tedesca di questo saggio è apparsa in Archiv für das Studium der neueren Sprachen und Literaturen 25 2.I (2015), pp. 83-I04. 